\title{
Isolated adrenal metastases of papillary thyroid carcinoma
}

\author{
Joana Maciel', Helena Vilar', Teresa C. Ferreira' ${ }^{2}$, Valeriano Leite ${ }^{1,3}$ \\ ${ }^{1}$ Endocrinology Department, Instituto Português de Oncologia de Lisboa Francisco Gentil, Lisbon, Portugal \\ ${ }^{2}$ Nuclear Medicine Department, Instituto Português de Oncologia de Lisboa Francisco Gentil, Lisbon, Portugal \\ ${ }^{3}$ Nova Medical School, New University of Lisbon, Lisbon, Portugal
}

Key words: thyroid cancer; papillary thyroid carcinoma; poorly differentiated thyroid carcinoma; adrenal metastases

Papillary thyroid cancer (PTC) usually runs an indolent clinical course, spreading to the regional lymph nodes (LN) and, less frequently, to the lungs or bones [1]. Distant metastases are found in less than $5 \%$ of patients at initial diagnosis and are associated with poor prognosis [2]. Adrenal metastates (AMs) in this setting are rare and usually associated with other distant metastases [3].

To our knowledge, there have been very few reported cases of PTC with metastatic spread only to the adrenal gland $[1,2,4,5]$. None of these cases achieved remission previously to the diagnosis of AMs. Herein, we report the first case of a patient with a PTC, who developed AMs after a period of complete remission.

A 68-year-old man underwent total thyroidectomy and left cervical lymphadenectomy (September 2015), following PTC diagnosis by fine-needle biopsy of a left thyroid nodule.

Pathology revealed a mixed classic and follicular variant of PTC with the largest diameter of $48 \mathrm{~mm}$ with extensive angioinvasion and cervical LN metastases. The first treatment with $3.7 \mathrm{GBq}(100 \mathrm{mCi})$ of radioactive iodine (RAI) under recombinant TSH (rhTSH) was performed (February 2016). The stimulated serum thyroglobulin (Tg) was $22.6 \mathrm{ng} / \mathrm{mL}$, and the whole-body scan (WBS) performed 48 hours after RAI treatment only revealed slight neck uptake. Serum $\mathrm{Tg}$ was $9 \mathrm{ng} / \mathrm{mL}$ 3 months after treatment, which gradually increased to $19 \mathrm{mg} / \mathrm{mL}$ (TSH $<0.02 \mu \mathrm{UL} / \mathrm{mL}$ ) until March 2017, when he underwent a right cervical LN dissection due to recurrent LN disease. Pathology showed LN metastases of PTC. Two months after this surgery, serum Tg was $17 \mathrm{ng} / \mathrm{mL}(\mathrm{TSH}<0.02 \mu \mathrm{UI} / \mathrm{mL})$.

A second RAI treatment with $3.7 \mathrm{GBq}(100 \mathrm{mCi})$ and rhTSH was administered (May 2017), with stimulated Tg of $53.5 \mathrm{ng} / \mathrm{mL}$ and WBS showing no abnormal uptake. Serum Tg level 3 months after treatment was 22 $\mathrm{ng} / \mathrm{mL}$, which increased in the following months to 2138 $\mathrm{ng} / \mathrm{mL}$. A left cervical LN recurrence was detected (Fig. 1) and he was submitted to a left neck dissection in August 2018. Pathology confirmed 4 LN metastases of PTC.

Three months after surgery (November 2018) he showed no evidence of disease, with an undetectable level of $\mathrm{Tg}$ and negative anti-Tg antibodies, which were maintained for the next 26 months, with normal neck imaging by ultrasound.

In January 2021, serum Tg increased from undetectable to $1758 \mathrm{ng} / \mathrm{mL}$. In this context, an FDG (2-[18F] fluoro-2deoxy-D-glucose) - PET (positron emission tomography) - CT (computed tomography) scan was requested, which showed abnormal uptake in the left adrenal gland (Fig. 2).

Left adrenalectomy was performed (March 2021) and a completely excised $100 \times 80 \times 50 \mathrm{~mm}$ AM of poorly differentiated thyroid carcinoma, with solid and insular pattern [stained positive for thyroid transcription factor 1 (TTF1) and Tg] was confirmed upon pathological examination (Fig. 3).

One month after surgery, the serum Tg level dropped to $24 \mathrm{ng} / \mathrm{mL}$ (TSH $<0.02 \mu \mathrm{UL} / \mathrm{mL}$ ).

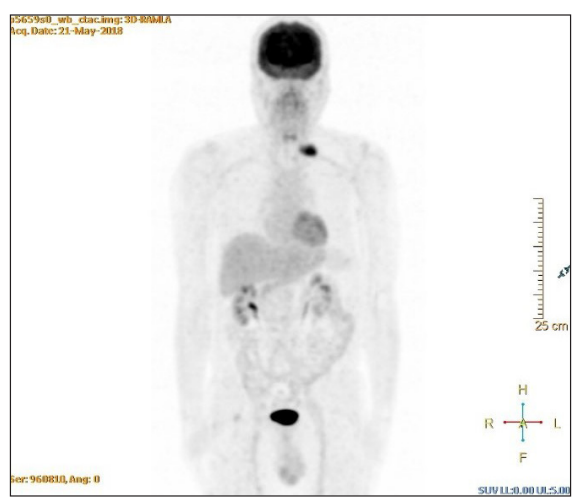

Figure 1. FDG/PET-CT (May/2018): Left supraclavicular lymph node with $40 \mathrm{~mm}$ high FDG uptake (SUVmax 10.5) 


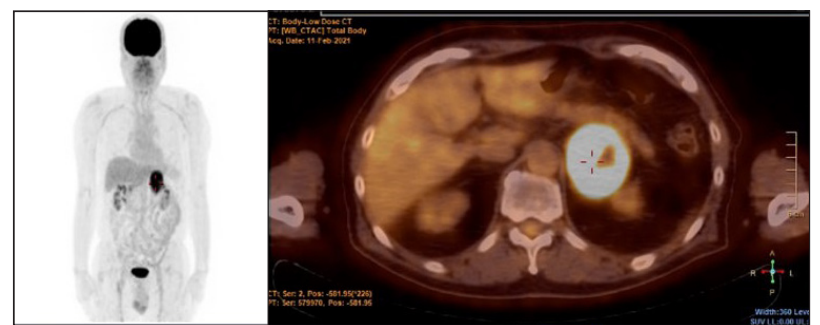

Figure 2. FDG/PET-CT (February/2021): left adrenal lesion with high FDG uptake (SUVmax 15.4). No anomalous uptake in other sites

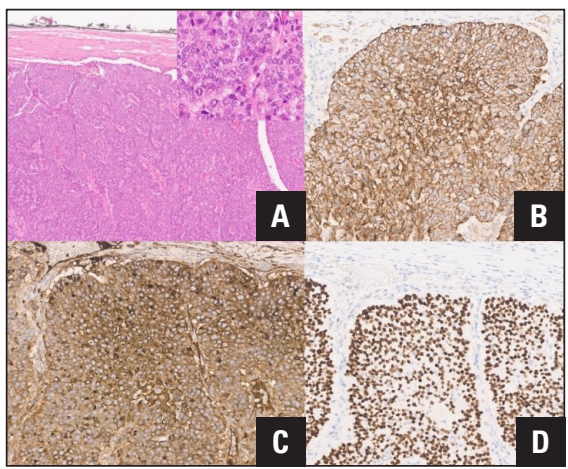

Figure 3. A. Haematoxylin and eosin staining of the adrenal metastasis with the characteristic nuclear features of PTC (inset); $B$. Cytokeratin AE1/3; C. Thyroglobulin; D. TTF1

The patient underwent a third RAI treatment with $5.5 \mathrm{GBq}(150 \mathrm{mCi})$ under rhTSH in June 2021. The post-therapy WBS showed no evidence of avid iodine disease. However, the FDG-PET-CT scan performed simultaneously with the RAI treatment showed a suspicious uptake in the right adrenal with SUVmax of 6.4. Stimulated Tg was $177 \mathrm{ng} / \mathrm{mL}$.

A thoracoabdominal-pelvic CT confirmed a right adrenal lesion of $18 \times 18 \times 13 \mathrm{~mm}$ with no evidence of disease at the left adrenalectomy bed (Fig. 4). Right adrenalectomy was then performed (August 2021), and pathology confirmed an AM of poorly differentiated thyroid carcinoma, TTF1+, and Tg + with a similar pattern of the left AM.

Fifteen days after this surgery serum $\mathrm{Tg}$ dropped to $12.5 \mathrm{ng} / \mathrm{mL}$ (TSH $0.11 \mu \mathrm{UI} / \mathrm{mL}$, negative anti-Tg antibodies). The patient is now under active surveillance.

AMs account for approximately $1.7 \%$ of DTC distant metastases and are mostly associated with the presence of other distant metastases [3]. To our knowledge there were only 4 reported cases of isolated AMs from PTC $[1,2,4,5]$. Our case is unique because AMs occurred after a significant period of complete remission. The increase in serum Tg was the initial clue to the diagnosis and the FDG uptake raised the suspicion of the lesions. Interestingly, serum Tg increased despite the poorly differentiated pattern of the AM.

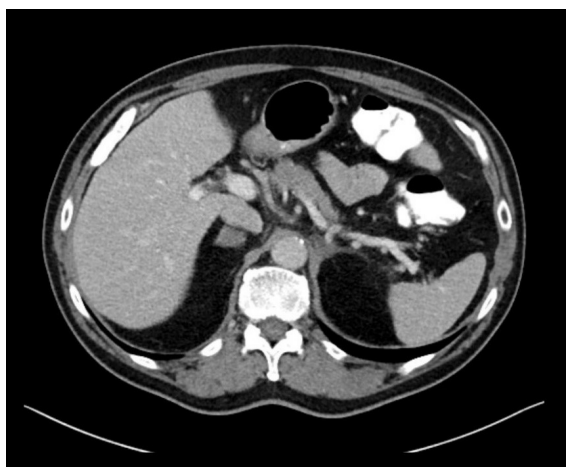

Figure 4. Abdominal-CT (July/2021): Right adrenal lesion of $18 \times$ $13 \times 18 \mathrm{~mm}$, with absolute washout of $45.5 \%$ and relative washout of $20 \%$ (indeterminate lesion)

This case underlines the importance of a close and prolonged surveillance of patients with high-risk thyroid cancer. The early diagnosis of distant metastasis can have a significant prognostic impact, making surveillance either with tumour markers or imaging procedures mandatory.

\section{Conflict of interests}

The authors have no conflicts of interest to disclose

\section{Statement of ethics}

The research was conducted ethically in accordance with the World Medical Association Declaration of Helsinki. Data were collected retrospectively.

\section{Competing interests}

None declared.

\section{Funding}

No funding was available.

\section{Acknowledgments}

The authors thank the Pathology Department of Instituto Português de Oncologia de Lisboa Francisco Gentil for providing representative images of the histological slides.

\section{References}

1. Wagenaar N, Oosterhuis JW, Rozendaal L, et al. Adrenal metastasis from a primary papillary thyroid carcinoma. Intern Med. 2008; 47(24): 2165-2168, doi: 10.2169/internalmedicine.47.1582, indexed in Pubmed: 19075543.

2. Koutkia P, Safer JD. Adrenal metastasis secondary to papillary thyroid carcinoma. Thyroid. 2001; 11(11): 1077-1079, doi: 10.1089/105072501753 271798, indexed in Pubmed: 11762719.

3. Batawil N. Papillary thyroid cancer with bilateral adrenal metastases. Thyroid. 2013; 23(12): 1651-1654, doi: 10.1089/thy.2013.0065, indexed in Pubmed: 23683128.

4. Praw SS, Ahmadi S, Martinez D. Papillary Thyroid Carcinoma with an Isolated Adrenal Metastasis. Proceedings of UCLA Healthcare. 2013; 17.

5. Blažeković I, Jukić T, Granić R, et al. An Unusual Case of Papillary Thyroid Carcinoma Iodine-131 Avid Metastasis to the Adrenal Gland. Acta Clin Croat. 2018; 57(2): 372-376, doi: 10.20471/acc.2018.57.02.20, indexed in Pubmed: 30431733. 